\title{
EXPERIMENTAL STUDIES OF THE DISSOCIATIVE RECOMBINATION PROCESSES FOR THE $\mathrm{C}_{6} \mathrm{D}_{6}^{+}$AND $\mathrm{C}_{6} \mathrm{D}_{7}^{+}$ IONS
}

M. Hamberg ${ }^{1}$, E. Vigren ${ }^{1}$, R.D. Thomas $^{1}$, V. Zhaunerchyk ${ }^{2}$, M. Zhang ${ }^{3}$, S. Trippel ${ }^{4}$, M. Kaminska ${ }^{5}$, I. Kashperka ${ }^{1}$, M. af Ugglas ${ }^{1}$, A. Källberg ${ }^{6}$, A. Simonsson ${ }^{6}$, A. Paál ${ }^{6}$, J. Semaniak ${ }^{5}$, M. $\operatorname{Larsson}^{1}$ and W.D. Geppert ${ }^{1}$

\begin{abstract}
We have investigated the dissociative recombination (DR) of the $\mathrm{C}_{6} \mathrm{D}_{6}^{+}$and $\mathrm{C}_{6} \mathrm{D}_{7}^{+}$ions using the CRYRING heavy-ion storage ring at Stockholm University, Sweden. The dissociative recombination branching ratios were determined at minimal collision energy, showing that the DR of both ions was dominated by pathways keeping the carbon atoms together in one product. The absolute reaction cross sections for the titular ions are best fitted by $\sigma\left(\mathrm{E}_{\mathrm{cm}}[\mathrm{eV}]\right)=1.3 \pm 0.4 \times$ $10^{-15}\left(\mathrm{E}_{\mathrm{cm}}[\mathrm{eV}]\right)^{-1.19 \pm 0.02} \mathrm{~cm}^{2}\left(\mathrm{C}_{6} \mathrm{D}_{6}^{+}\right)$and $\sigma\left(\mathrm{E}_{\mathrm{cm}}[\mathrm{eV}]\right)=1.1 \pm 0.3 \times$ $10^{-15}\left(\mathrm{E}_{\mathrm{cm}}[\mathrm{eV}]\right)^{-1.33 \pm 0.02} \mathrm{~cm}^{2}\left(\mathrm{C}_{6} \mathrm{D}_{7}^{+}\right)$in the intervals $3-300 \mathrm{meV}$ and 3-200 meV respectively. The thermal rate constants of the titular reactions are best described by: $\mathrm{k}(\mathrm{T})=1.3 \pm 0.4 \times 10^{-6}(\mathrm{~T} / 300)^{-0.69 \pm 0.02}$ $\mathrm{cm}^{3} \mathrm{~s}^{-1}$ for $\mathrm{C}_{6} \mathrm{D}_{6}^{+}$and $\mathrm{k}(\mathrm{T})=2.0 \pm 0.6 \times 10^{-6}(\mathrm{~T} / 300)^{-0.83 \pm 0.02} \mathrm{~cm}^{3} \mathrm{~s}^{-1}$ for $\mathrm{C}_{6} \mathrm{D}_{7}^{+}$. These expressions correlates well with earlier flowing afterglow studies on the same process.
\end{abstract}

\section{Introduction}

Polyaromatic hydrocarbons (PAHs) are thought to play a central role in astrochemistry. It is suggested that PAHs may contain up to $20 \%$ of the available carbon

\footnotetext{
1 Department of Physics, Stockholm University, SE-106 91 Stockholm, Sweden; e-mail: mathias.hamberg@physto.se

2 Radboud University Nijmegen, Institute for Molecules and Materials, PO Box 9010, 6500 GL Nijmegen, The Netherlands

3 Institute of Modern Physics, Chinese Academy of Sciences, 509 Nanchang Rd., Lanzhou 730000, China

4 Department of Physics, Albert-Ludwigs Universität Freiburg, Hermann-Herder-Str. 3, 79104 Freiburg, Germany

5 Jan Kochanowski University, Świȩtokrzyska 15, 25406 Kielce, Poland

6 Manne Siegbahn Laboratory, Frescativägen 26, 11418 Stockholm, Sweden
} 
in the ISM (Boulanger 1999). Whereas there is strong evidence for the presence of PAHs in various astronomical objects, only the simplest aromatic compound, benzene $\left(\mathrm{C}_{6} \mathrm{H}_{6}\right)$ has been unambiguously detected by the Infrared Space Observatory in the protoplanetary nebula CRL 618 (Cernicharo et al. 2001). Furthermore, benzene is present in the atmospheres of gaseous giant planets and has been found in Titan's atmosphere by the Composite Infra-Red spectrometer (CIRS) on board the Cassini-Huygens mission (Vinatier et al. 2007).

Benzene can function as template in the build-up of higher hydrocarbons including PAHs in the interstellar medium. In a recent paper by Le Page et al. (2009) it is suggested that PAHs may play a key role as catalyst in forming molecular hydrogen in the interstellar medium. This involves chemical trapping of $\mathrm{H}$ atoms on dehydrogenated PAHs, ionisation of the PAH, followed by addition of another $\mathrm{H}$ atom with a subsequent release of $\mathrm{H}_{2}$ through dissociative recombination (DR). This sequence constitutes a circular processes in which a hydrogen molecule is formed from two atoms and the dehydrogenated $\mathrm{PAH}$ is recycled. Le Page et al. (2009) assume in their model branching fractions of $50 \%$ each for the two reaction channels leading to $\mathrm{H}$ and $\mathrm{H}_{2}$ respectively in the $\mathrm{DR}$ of $\mathrm{C}_{6} \mathrm{H}_{7}^{+}$.

Investigation of the synthesis for benzene itself in these environments is therefore a key issue. In interstellar clouds as well as Titan's atmosphere dissociative recombination of the $\mathrm{C}_{6} \mathrm{H}_{7}^{+}$ion (which is formed by a sequence iof ion-neutral reactions) leading to the unprotonated benzene has been invoked as the final step in the synthesis of this compound (Woods et al. 2002; Vuitton et al. 2008):

$$
\mathrm{C}_{6} \mathrm{H}_{7}^{+}+\mathrm{e}^{-} \rightarrow \mathrm{C}_{6} \mathrm{H}_{6}+\mathrm{H}
$$

The DR process itself is, however, highly exoergic $(\Delta \mathrm{H}=-5.73 \mathrm{eV}$ for the abovementioned reaction) and other reaction channels including ejection of carboncontaining fragments may be feasible. In addition, it has recently been shown that ejection of a single $\mathrm{H}$ atom can be an only minor reaction channel for protonated molecules (Geppert et al. 2005; Hamberg 2010). Therefore knowledge of the branching ratio of the DR of protonated benzene is crucial to validate the postulated formation mechanism in the interstellar medium and planetary atmospheres. This Paper presents an experimental study into the DR rate constant and product branching fractions of $\mathrm{C}_{6} \mathrm{D}_{6}^{+}$and $\mathrm{C}_{6} \mathrm{D}_{7}^{+}$.

\section{Experiment}

The experiments were carried out at the heavy-ion storage ring CRYRING at Stockholm University, Sweden. The procedure has been explained in detail previously (Neau et al. 2000) and will therefore only be summarised here. The ions were formed by a discharge in a mixture of fully deuterated benzene and deuterium gas in a hollow cathode type ion source. The gas was pulsed into the ion source reaching a peak pressure of $\sim 1$ Torr in order to strive for collisional quenching. The ions were then accelerated by $40 \mathrm{kV}$ and mass selected by the first bending 
magnet in the injection line. After a 10-turn multi injection procedure into the storage ring the ions were further accelerated by a RF-system to the maximum kinetic energy of $\sim 96 / \mathrm{m}_{\text {ion }} \mathrm{MeV}$ (where $\mathrm{m}_{\text {ion }}$ is the ion mass in amu). A long storage time of several seconds was ensured due to the low ring pressure of residual gas (typically $\sim 10^{-11}$ Torr). Thus the ions could be left to de-excite for 3 seconds before the measurement sequence started. This left ample time for vibrationally excited inner degrees of freedom to relax. In an electron cooler a cold, monoenergetic electron beam was overlapping the path of the ions over $\sim 85 \mathrm{~cm}$ which served as DR interaction region. Neutral particles formed in the process were separated from the ion beam, since they are unaffected by the dipole magnet located after the electron cooler and were recorded by an energy sensitive ion implanted silicon detector (IISD).

\section{Data analysis and results}

\subsection{Branching fractions}

Branching fraction measurements were performed at $0 \mathrm{eV}$ nominal relative kinetic energy. Neutral fragments deriving from DR-events impinge on the IISD at a timescale (ns) much shorter than the shaping time of the preamplifier $(\mu \mathrm{s})$. Therefore the detected signal for each event will essentially reflect the sum of the masses of all the incoming fragments and thus yield no information about the branching ratio. To come around this problem a transparent metal grid with a transmission probability of $0.297 \pm 0.015$ is inserted in front of the IISD. With the grid inserted all fragment masses appear in the energy spectrum and branching ratios can be derived from the intensity distribution of the different mass signals. Fragment energy spectra with and without grid for $\mathrm{C}_{6} \mathrm{D}_{6}^{+}$ion are shown in Figure 1. One can see a small signal around energy channel 950 indicating that some fragments containing $3 \mathrm{C}$-atoms are breaking loose from the rest of the molecule although the majority of DR processes seem to keep all six carbon atoms together. If splitting up of the ions into 2 fragments containing 3 carbon atoms would dominate the reaction flux the spectra measured with the grid inserted would be severely different from the ones recorded without grid. The peak at full energy would then be smaller than the 3C peak found around channel 950 due to the comparatively low transmissibility of the grid. No obvious peaks are found at the energies corresponding to fragnments with two or four carbon atoms. The lack of smaller fragments is even more pronounced for the $\mathrm{C}_{6} \mathrm{D}_{7}^{+}$ion where the spectrum structure essentially indicates that all fragments keep the six carbon atoms together and no other peaks are distinguishable (see Fig. 1). Rescaling the MCA-channels into mass units (which can only be done in a very approximative way due to the broadness of the features) shows that the difference of the peak centers for the peaks corresponding to the highest masses in Figure 1 with the grid in and out is in the order of one amu. This is well in line with ejection of one or two deuterium atoms and a preservation of the aromatic ring structure during 


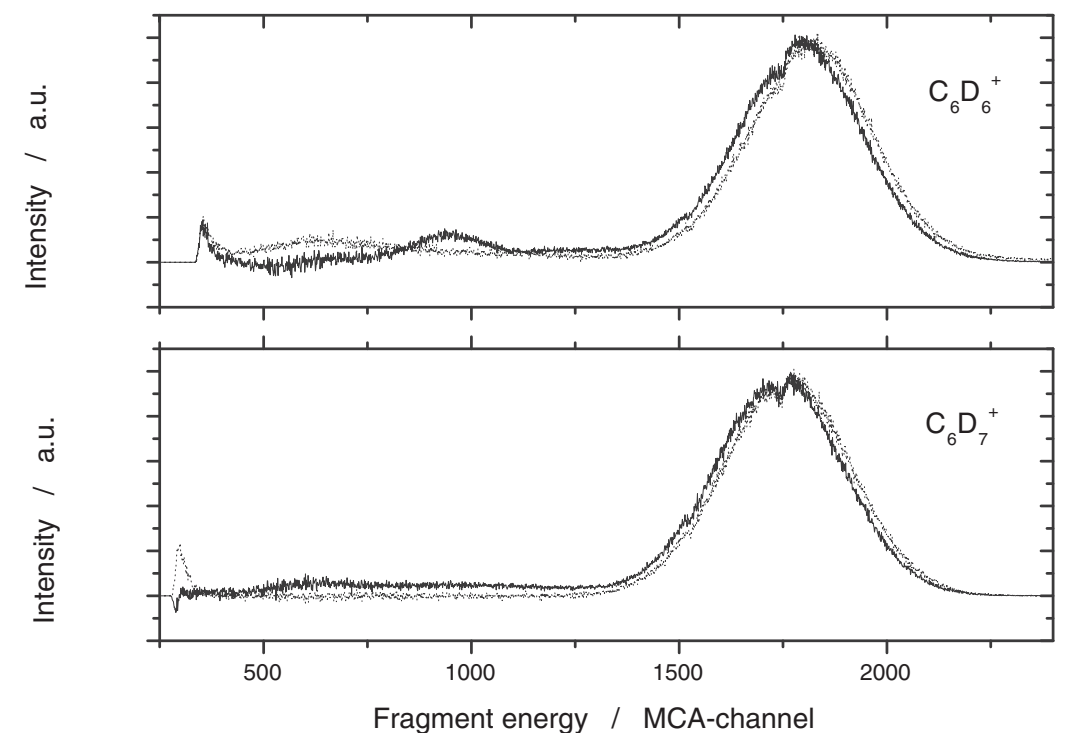

Fig. 1. MCA spectra from branching fraction measurements of the $\mathrm{C}_{6} \mathrm{D}_{6}^{+}$(top) and $\mathrm{C}_{6} \mathrm{D}_{7}^{+}$(bottom) ions. The data obtained without grid is depicted with a dotted line whereas the ones recorded with the grid inserted are represented with a solid line.

DR. However, further analysis were complicated due to losses of fast deuterium fragments bypassing the active detector area. Possible losses of heavier fragments containing carbon atoms were estimated to be well below $10 \%$ using Monte Carlo simulations in a similar fashion as Thomas (2008) and should therefore not affect the obtained spectra much.

\subsection{Cross section and rate constant}

To measure the DR rate constant the electron velocity was ramped relative to the ion velocity by scanning the electron acceleration voltage in the electron cooler. The neutral particles deriving from the DR-processes were detected by the IISD and counted by a multichannel scaler (MCS) using $2 \mathrm{~ms}$ time bins. Each bin corresponds to an interaction energy in the interval between $\sim 2-1000 \mathrm{meV}$. Background processes from ions interacting with residual gas in the storage ring were simultaneously collected by a micro channel plate (MCP) located at the end of a straight section and recorded by a MCS. The background signal intensity is directly proportional to the ion current and could therefore be scaled to the absolute ion current which was measured with a capacitive pick-up calibrated by an AC transformer directly after acceleration (Paal et al. 2006). Therefore the mean ion current throughout the ring-cycle could be determined and fitted by a decay curve. This fit was also used for background subtraction of the DR MCS spectrum before 
applying the following formula for calculation of the rate coefficient:

$$
k=\left(\frac{d N}{d t}\right) \frac{v_{i} v_{e} e^{2} r_{e}^{2} \pi}{I_{e} I_{i} l}
$$

where $d N / d t$ is the measured DR-count rate, $I_{e}, v_{e}$ and $I_{i}, v_{i}$ are the electron and ion currents and velocities (in the laboratory frame), respectively and $e$ is the elementary charge, $r_{e}$ is the radius of the electron beam and $l$ is the interaction region length.

Corrections to the data had to be made for: (i) Space charge effects where the electrons experience a drop in the acceleration potential at the cathode in the cooler due to free electrons traveling ahead of them. (ii) Toroidal effects deriving from ion electron collisions at higher energies than the detuning energy in the regions where the electron beam is bent into and out from the interaction region. (iii) The electrons have a non negligible transversal energy spread of $2 \mathrm{meV}$. The rate constant can therefore be expressed as below through which the DR cross section can be deconvoluted using Fourier methods (Mowat et al. 1995):

$$
k=\int_{0}^{\infty} v_{r e l} f\left(v_{\perp}\right) \sigma\left(v_{r e l}\right) d v_{\perp}
$$

where $f\left(v_{\perp}\right)$ is the transversal electron velocity distribution in the center of mass frame, $v_{\perp}$ the transversal electron velocity and $v_{r e l}$ the relative velocity respectively. The result for the $\mathrm{C}_{6} \mathrm{D}_{6}$ ion is displayed in Figure 2 together with the best fit between 3-300 meV giving a cross section of $\sigma\left(\mathrm{E}_{\mathrm{cm}}[\mathrm{eV}]\right)=1.3 \pm 0.4 \times 10^{-15}$ $\left(\mathrm{E}_{\mathrm{cm}}[\mathrm{eV}]\right)^{-1.19 \pm 0.02} \mathrm{~cm}^{2}$. Applying the same procedure for the $\mathrm{C}_{6} \mathrm{D}_{7}^{+}$ion (see Fig. 2) resulted in a best fit over the interval 3-200 meV of: $\sigma\left(\mathrm{E}_{\mathrm{cm}}[\mathrm{eV}]\right)=1.1 \pm$ $0.3 \times 10^{-15}\left(\mathrm{E}_{\mathrm{cm}}[\mathrm{eV}]\right)^{-1.33 \pm 0.02} \mathrm{~cm}^{2}$. The thermal rate constant can be obtained from the cross sections by applying the formula:

$$
k(T)=\frac{8 \pi m_{e}}{\left(2 \pi m_{e} k_{B} T\right)^{3 / 2}} \int_{0}^{\infty} E_{c m} \sigma\left(E_{c m}\right) e^{-E_{c m} / k_{B} T} d E_{c m},
$$

where $m_{e}$ is the electron mass, $k_{B}$ is Boltzmann's constant, $T$ is the electron temperature and $\mathrm{E}_{c m}$ is the centre of mass energy. The obtained thermal rates are: $\mathrm{k}(\mathrm{T})=1.3 \pm 0.4 \times 10^{-6}(\mathrm{~T} / 300)^{-0.69 \pm 0.02} \mathrm{~cm}^{3} \mathrm{~s}^{-1}$ for $\mathrm{C}_{6} \mathrm{D}_{6}^{+}$and $\mathrm{k}(\mathrm{T})=2.0 \pm$ $0.6 \times 10^{-6}(\mathrm{~T} / 300)^{-0.83 \pm 0.02} \mathrm{~cm}^{3} \mathrm{~s}^{-1}$ for $\mathrm{C}_{6} \mathrm{D}_{7}^{+}$. The systematical errors are arising from uncertainties in the ion and electron currents and the length of the interaction region electron density. These errors combined with the statistical uncertainties are estimated to be $25 \%$ and $30 \%$ for $\mathrm{C}_{6} \mathrm{D}_{6}^{+}$and $\mathrm{C}_{6} \mathrm{D}_{7}^{+}$respectively.

\section{Discussion}

For both ions DR experiments have previously been performed using flowing afterglow (FA) technique. The thermal rates at $300 \mathrm{~K}$ was found to be $1.0 \pm 0.3 \times$ $10^{-6} \mathrm{~cm}^{3} \mathrm{~s}^{-1}\left(\mathrm{C}_{6} \mathrm{D}_{6}^{+}\right)$and $2.4 \pm 0.4 \times 10^{-6} \mathrm{~cm}^{3} \mathrm{~s}^{-1}\left(\mathrm{C}_{6} \mathrm{D}_{7}^{+}\right)$, which is in very good 


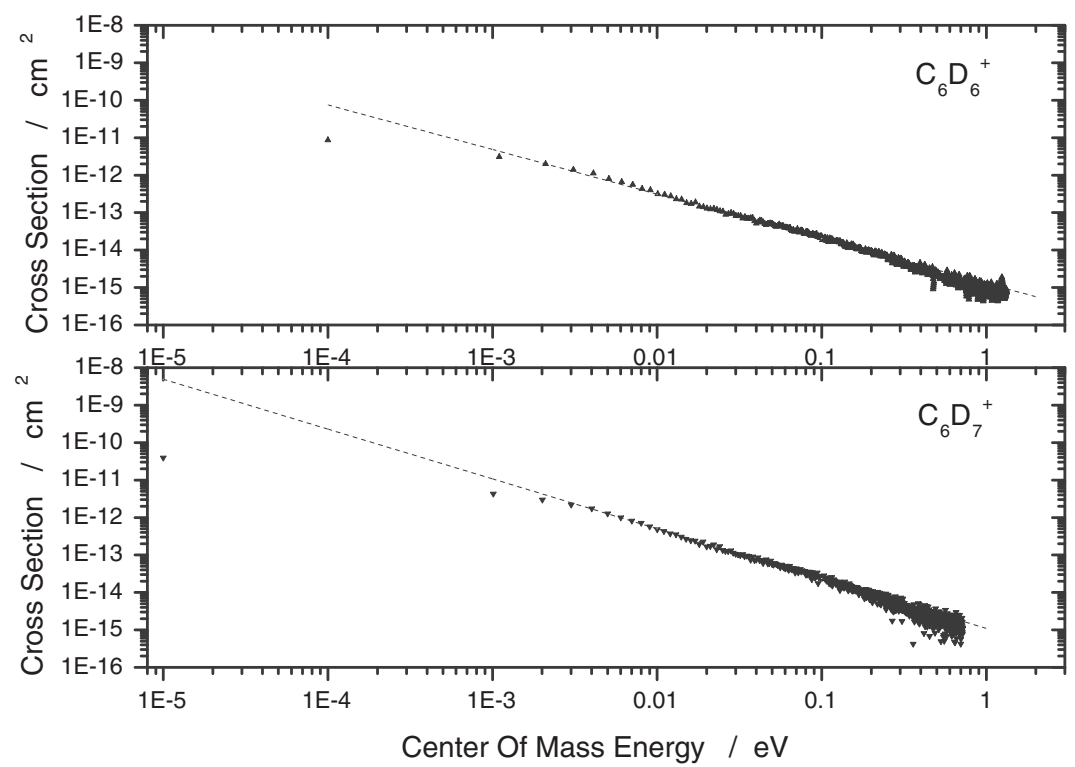

Fig. 2. Cross section of the $\mathrm{DR}$ of $\mathrm{C}_{6} \mathrm{D}_{6}^{+}$(top) and $\mathrm{C}_{6} \mathrm{D}_{7}^{+}$(bottom) versus relative kinetic energy. The triangles shows individually measured spots while the dashed line shows the best fit between 3-300 meV $\left(\mathrm{C}_{6} \mathrm{D}_{6}^{+}\right)$and 3-200 $\mathrm{meV}\left(\mathrm{C}_{6} \mathrm{D}_{7}^{+}\right)$respectively.

agreement with previous results for the undeuterated isotopomers (Abouelaziz et al. 1993; McLain et al. 2004). The lowest formation enthalpy for the undeuterated $\mathrm{C}_{6} \mathrm{H}_{6}^{+}$and $\mathrm{C}_{6} \mathrm{H}_{7}^{+}$ions are found for their cyclic forms and are $975.8 \mathrm{~kJ} \mathrm{~mol}^{-1}$ and $854 \mathrm{~kJ} \mathrm{~mol}^{-1}$ respectively (Lias et al. 1988). Abouelaziz et al. (1993) found in their DR experiment of $\mathrm{C}_{6} \mathrm{H}_{6}^{+}$no difference in the results when they formed the ion through charge transfer reaction with $\mathrm{C}^{+}, \mathrm{Xe}^{+}$and $\mathrm{Kr}^{+}$suggesting that the structures of the product ions were the same in each case. These charge transfer processes are exoergic with $2 \mathrm{eV}, 2.88 \mathrm{eV}$ and $4.75 \mathrm{eV}$ respectively, which can be compared to at least $2.8 \mathrm{eV}$ needed for breaking the benzene ring. Even at higher exoergicities ring opening seems therefore not to occur. In the ion source the $\mathrm{C}_{6} \mathrm{D}_{6}^{+}$ions could be formed through charge transfer from $\mathrm{D}_{2}^{+}$and $\mathrm{D}^{+}$(exoergic with $6.17 \mathrm{eV}$ and $4.34 \mathrm{eV}$, respectively (Lias et al. 1988)) or charge transfer reaction with $\mathrm{D}_{3}^{+}$leading to $\mathrm{D}_{2}$ and $\mathrm{D}$ (close to thermoneutral, i.e. with a reaction enthalpy close to zero). Electron impact and Penning ionization are other processes contributing and one can not rule out formation of other isomers. Nevertheless, we assume that the cyclic structure should dominate the stored ions. In all these thermodynamic considerations the enthalpies of the undeuterated compounds were considered due to the lack of data for the heavier isotopomeres.

In our set-up the $\mathrm{C}_{6} \mathrm{D}_{7}^{+}$ions should predominantly be formed through protonation by $\mathrm{D}_{3}^{+}$and to some extent reactions with $\mathrm{D}_{2}^{+}$and $\mathrm{D}^{+}$(exoergic with $3.48 \mathrm{eV}$, 
$5.17 \mathrm{eV}$ and $7.87 \mathrm{eV}$ respectively, Lias \& Ausloos 1985). It is also found that the ion can be formed by hydrogen atom abstraction from hydrogen donors. Flowing afterglow experiments were carried out by Milligan et al. (2002) and Spanel et al. (1995) in which they found that reaction of $\mathrm{H}_{3}^{+}$with benzene leads to $\mathrm{C}_{6} \mathrm{H}_{7}^{+}$with unity probability which was described as "a manifestation of the extraodrinary stability of this protonated molecule". Unfortunately however, their type of setup was not sensitive to structural isomers (Spanel \& Smith 1998). This is somewhat disadvantageous, since also stable five membered ring and open-chain isomers of $\mathrm{C}_{6} \mathrm{D}_{7}^{+}$exist. Kuck (1990) claimed that the cyclic $\mathrm{C}_{6} \mathrm{H}_{7}^{+}$was, beyond any doubt, the most stable light hydrogen isomer. In a ICR study, even reactions between aliphatic (neutrals e.g. $\left.\mathrm{C}_{3} \mathrm{H}_{4}\right)$ and ions $\left(\mathrm{C}_{3} \mathrm{H}_{4}^{+}\right)$resulting in the formation of $\mathrm{C}_{6} \mathrm{H}_{7}^{+}$ at least partly lead to protonated cyclobenzene (Lias \& Ausloos 1985). This shows that even if the formation process of $\mathrm{C}_{6} \mathrm{D}_{7}^{+}$in our ion source to some extent results in open-chain $\mathrm{C}_{6} \mathrm{D}_{7}^{+}$isomers, ring closure of these species to deuteronated benzene could be possible. Although we cannot exclude different isomeric forms we find it likely that the aromatic form should be dominating. In addition, it should be noted that even protonated benzene has two isomers - a $\sigma$ and a $\pi$-complex. However, Solca \& Dopfer (2002) found no presence of the $\pi$-complex in a spectroscopic study of $\mathrm{C}_{6} \mathrm{H}_{7}^{+}$ions produced by proton transfer to benzene from $\mathrm{H}_{3}^{+}$. This is probably also the major formation process of the $\mathrm{C}_{6} \mathrm{H}_{7}^{+}$ion in our experiment, thus we assume the ion present in our experiment to be the $\sigma-\mathrm{C}_{6} \mathrm{H}_{7}^{+}$.

Thus, our findings are in line with a formation of benzene in the interstellar medium, circumstellar envelopes and planetary ionospheres through ion-neutral reactions leading to $\mathrm{C}_{6} \mathrm{H}_{7}^{+}$followed by DR yielding benzene. Also the rate constant previously measured in flowing afterglow experiments (Abouelaziz et al. 1993; McLain et al. 2004) and used in models of Titan's ionosphere (Vuitton et al. 2008) has been corroborated in our study, albeit the temperature dependence of the DR rate of $\mathrm{C}_{6} \mathrm{D}_{7}^{+}$has been found to be considerably weaker (the exponential factor being -0.83 instead of -1.3 ). This could be due to a different reactivity of ground and rovibrationally excited states of the ion. In our storage ring, as opposed to afterglow experiments, the distribution between these states is not changed throughout the energy scan. Such different exponential factors of temperature dependence lead to considerably unlike rates at very low temperatures and it has to be seen how model predictions of benzene abundances in very cold environments like dark clouds are affected by that. The effect of the new rate constants on models of warmer environments like planetary ionospheres are, however, probably negligible.

Our results on the $\mathrm{DR}$ of $\mathrm{C}_{6} \mathrm{D}_{7}^{+}$pose a challenge to neither the invoked formation mechanism of benzene nor to the proposed $\mathrm{H}_{2}$ formation catalyzed by PAHs. They can, on the contrary, not serve to corroborate them, since we could not establish the branching ratio of the ejection of $\mathrm{H}_{2}$ and the formation of benzene. If this reaction would only result in production of $\mathrm{H}_{2}$, it would render the invoked reaction sequence of benzene formation unfeasible. If on the other hand the branching ratio of the formation of benzene would be close to 1 , the catalytic cycle of $\mathrm{H}_{2}$ formation postulated by Le Page et al. (2009) would be impossible. Experiments 
on storage rings with higher rigidity, which would enable us to distinguish between $\mathrm{H}_{2}$ and $\mathrm{H}$ ejection are therefore indespensable to clarify the validity of those two reaction sequences.

\section{Conclusion}

Storage ring technique has for the first time been employed for DR measurements of the simplest aromatic hydrocarbon ions $\mathrm{C}_{6} \mathrm{D}_{6}^{+}$and $\mathrm{C}_{6} \mathrm{D}_{7}^{+}$. The rate constants are in line with previous flowing afterglow measurements at $300 \mathrm{~K}$. Although it was not possible to fully elucidate the branching fractions between the reaction channels, our studies showed that the $\mathrm{C}_{6}$-structure is retained almost entirely in both cases. Dissociative recombination of $\mathrm{C}_{6} \mathrm{H}_{7}^{+}$therefore still can be regarded as a feasible final step in the synthesis of benzene the interstellar medium and planetary ionospheres. Our findings also do not disprove the molecular hydrogen formation mechanism by PAHs proposed by Le Page et al. (2009).

We thank the staff at the Manne Siegbahn Laboratory for making these experiments possible by excellent technical support. W.D.G. thanks the Swedish Research Council for his Senior Researcher grant (contract number 2006-427) and the Swedish Space Board (grant number 76/06). M.K. thanks the Swedish Institute for financial support and also acknowledges support from the Ministry of Science and Higher Education, Poland, under contract N202 111 31/1194. S.T. acknowledges financing from the COST ACTION (CM0805).

\section{References}

Abouelaziz, H., Gomet, J.C., Pasquerault, D., Rowe, B.R., \& Mitchell, J.B.A., 1993, J. Chem. Phys., 99, 237

Boulanger, F., 1999, in Solid State Matter: The ISO Revolution, Lecture 2, ed. L. dH́endecour, C. Joblin, \& A. Jones (New York Springer), 19

Cernicharo, J., Heras, A.M., Tielens, A.G.G.M., et al., 2001, Astrophys. J. Lett., 546, 123

Geppert, W.D., Thomas, R.D., Ehlerding, A., et al., 2005, J. Phys. Conf. Ser., 4, 26

Hamberg, M., 2010, A\&A, 514, A83

Kuck, D., 1990, Mass Spectrometry Reviews, 9, 187

Le Page, V., Snow, T.P., \& Bierbaum, V.M., 2009, ApJ, 704, 274

Lias, S.G., \& Ausloos, P., 1985, J. Chem. Phys., 82, 3613

Lias, S.G., Bartmess, J.E., Liebman, J.F., et al., 1988, J. Phys. Chem. Ref. Data., 17 Suppl. 1

McLain, J.L., Poterya, V., Molek, C.D., Babcock, L.M., \& Adams, N.G., 2004, J. Phys. Chem., 108, 6704

Milligan, D.B., Wilson, P.F., Freeman, C.G., Meot-Ner, M., \& McEwan, M.J., 2002, J. Phys. Chem. A, 106, 9745

Mowat, J.R., Danared, H., Sundtroem, G., et al., 1995, Phys. Rev. Lett., 74, 50 
Neau, A., Al-Khalili, A., Rosén, S., et al., 2000, J. Chem. Phys., 113, 1762

Paal, A., Simonsson, A., Dietrich, J., \& Mohos, I., 2006, Proceedings of EPAC2006, 1196

Solca, N., \& Dopfer, O., 2002, Angew. Chem. Int. Ed., 41, 3628

Spanel, P., \& Smith, D., 1998, Int. J. Mass Spectrom., 181, 1

Spanel, P., Smith, D., \& Henchman, M., 1995, Int. J. Mass Spectrom. Ion Proc., 141, 117

Thomas, R.D., 2008, Mass Spectrom. Rev., 27, 485

Vinatier, S., Bézard, B., Fouchet, T., et al., 2007, Icarus, 188, 120

Vuitton, V., Yelle, R., \& Cui, J., 2008, J. Geophys. Res., 113, E05007

Woods, P.M., Millar, T.J., Zijlstra, A.A., \& Herbst, E., 2002, Astrophys J. Lett., 574, 167 
